ORIGINAL ARTICLE

\title{
Early onset neonatal meningitis in Australia and New Zealand, 1992-2002
}

\section{May, A J Daley, S Donath, D Isaacs, on behalf of the Australasian Study Group for Neonatal Infections}

See end of article for authors' affiliations

Correspondence to:

Professor Isaacs,

Department of

Immunology and Infectious

Diseases, The Children's

Hospital at Westmead,

Locked Bag 4001,

Westmead, NSW 2145,

Australia; davidi@chw.

edu.au

Accepted 8 February 2005

Published Online First

5 May 2005

Objectives: To study the epidemiology of early onset neonatal bacterial meningitis (EONBM) in Australasia.

Design: Prospective surveillance study, 1992-2002, in 20 neonatal units in Australia and New Zealand. EONBM was defined as meningitis occurring within 48 hours of delivery.

Results: There were 852 babies with early onset sepsis, of whom 78 (9.2\%) had EONBM. The incidence of early onset group B streptococcal meningitis fell significantly from a peak of $0.24 / 1000$ live births in 1993 to $0.03 / 1000$ in 2002 ( $p$ trend $=0.002$ ). There was no significant change over time in the incidence of Escherichia coli meningitis. The rate of EONBM in very low birthweight babies was $1.09 / 1000$ compared with the rate in all infants of $0.11 / 1000$. The overall rate of EONBM was $0.41 / 1000$ in 1992 and 0.06 in 2001 , but this trend was not significant ( $p$ trend $=0.07$ ). Case-fatality rates for EONBM did not change significantly with time. Birth weight $<1500 \mathrm{~g}$ (odds ratio (OR) 7.2 (95\% confidence interval (Cl) 4.8 to 10.9)) and Gram negative bacillary meningitis (OR $3.3(95 \% \mathrm{Cl} 2.2$ to 4.9)) were significant risk factors for mortality. Sixty two percent of the 129 babies who died from early onset sepsis or suspected sepsis did not have a lumbar puncture performed.

Conclusion: The incidence of early onset group B streptococcal meningitis has fallen, probably because of maternal intrapartum antibiotic prophylaxis, without a corresponding change in $E$ coli meningitis. Gram negative bacillary meningitis still carries a worse prognosis than meningitis with a Gram positive organism.

A retrospective Australian study, 1987-1989, reported an incidence of neonatal meningitis of $0.17 / 1000$ live births. ${ }^{1}$ Data for 1992-1993 from the Australasian Study Group for Neonatal Infections (ASGNI) found that $23 \%$ of babies with early onset sepsis (less than 48 hours of age) had meningitis. ${ }^{2}$ Both these studies found the predominant organisms to be group B streptococcus (GBS) and Escherichia coli, a trend observed in other industrialised countries.

From the mid-1990s, the use of intrapartum prophylactic antibiotics in high risk pregnancies, or in women known to be GBS carriers, has been associated with a significant reduction in the amount of early onset neonatal sepsis due to GBS. ${ }^{4}$ In contrast, rates of early onset sepsis due to Gram negative organisms are reported as being mostly static over the last 10 years. ${ }^{5}$ In very low birthweight (VLBW, $<1500 \mathrm{~g}$ ) babies in the United States, however, E coli sepsis may have increased. ${ }^{7-9}$ It is not known what impact maternal intrapartum antibiotic prophylaxis has had on the epidemiology of neonatal meningitis, and in particular early onset neonatal bacterial meningitis (EONBM) in Australasia.

We present data collected prospectively by neonatologists since 1992 on newborns in hospital with systemic sepsis, and we concentrate on the epidemiology of EONBM. These data were collected during a period when maternal intrapartum antibiotic prophylaxis became "best practice" in Australasia, and therefore provides an opportunity to look for the same reduction in EONBM as seen in early onset neonatal sepsis.

\section{METHODS}

The ASGNI was formed in late 1991, with the aim of collecting clinically relevant data on neonatal infections. By 1997, 14 Australian neonatal units and two New Zealand units were participating. Eleven of the 14 Australian units are attached to maternity units, and include the largest neonatal unit in each major city in Australia. Overall the data include at least $10 \%$ of all births in Australia in the last 11 years.

From 1992 to 2002 inclusive, participating neonatologists recorded data on babies with sepsis (defined as septicaemia and/or meningitis) using a standard proforma which was returned to the ASGNI annually. Only data on babies who meet the following definitions of sepsis were collected. The data collected included birth weight and gestational age, indigenous status, age at time of sepsis, results of blood and cerebrospinal fluid (CSF) cultures and associated sites of infection. Deaths were recorded as being directly related to, possibly related to, or unrelated to sepsis. Invasive neonatal sepsis was defined as a case with clinical symptoms and supportive laboratory evidence of sepsis and a positive culture of a single potential pathogen from blood and/or CSF. Clinical symptoms include fever or temperature instability, irritability, apnoea and/or bradycardia, or other clinical findings judged by the treating physician to be consistent with a diagnosis of sepsis. Meningitis was defined as a clinical presentation consistent with meningitis and either a positive CSF culture of bacteria or a positive blood culture in association with an abnormal CSF white blood cell count $\left(>100 \times 10^{6} / 1\right)$. Babies with ventriculoperitoneal shunt infections were excluded. Neonatologists were asked not to report babies with positive cultures that were clinically suspected to be contaminants. No information is available on treatment of babies or maternal risk factors for GBS.

Abbreviations: ASGNI, Australasian Study Group for Neonatal Infections; $\mathrm{Cl}$, confidence interval; CSF, cerebrospinal fluid; EONBM, early onset neonatal bacterial meningitis; GBS, group B streptococcus; VLBW, very low birth weight 
Table 1 Organisms causing early onset neonatal bacterial meningitis, Australasia, 1992-2002

\begin{tabular}{lll}
\hline Organism & $\begin{array}{l}\text { Total No of } \\
\text { cases (\%) }\end{array}$ & $\begin{array}{l}\text { No of cases in } \\
\text { VLBW babies (\%) }\end{array}$ \\
\hline Group B streptococcus & $48(62)$ & $3(18)$ \\
Escherichia coli & $16(21)$ & $10(59)$ \\
Listeria monocytogenes & $3(4)$ & $1(6)$ \\
Enterococcus spp & $2(3)$ & $1(6)$ \\
Haemophilus influenzae (non-Hib) & $2(3)$ & $1(6)$ \\
Streptococcus pneumoniae & $2(3)$ & - \\
Enterobacter spp & $1(1)$ & - \\
Klebsiella spp & $1(1)$ & - \\
Neisseria meningitidis & $1(1)$ & - \\
Staphylococcus aureus & $1(1)$ & $1(6)$ \\
Pseudomonas aeruginosa & $1(1)$ & - \\
Total & 78 & 17 \\
\hline Hib, Haemophilus influenzae type b; & VLBW, very low birth weight. \\
\hline
\end{tabular}

Early onset sepsis was defined as sepsis within 48 hours of delivery. EONBM was defined as bacterial meningitis diagnosed from a CSF specimen collected within 48 hours of delivery. An analysis was also performed on infants with onset of meningitis at 3-7 days of age, because some authorities define early onset sepsis as infection occurring within seven days of birth. Incidence rates were calculated as the sum of the number of cases of early onset meningitis in babies born in reporting units divided by the sum of the number of live births in these units. Rates are expressed per 1000 live births. In 1992, 1993, and 2000, fewer units contributed data. To determine if the number of cases for these years was representative, the data from the two hospitals that contributed over the entire 11 year period were analysed separately. The rates of EONBM in VLBW infants were determined using estimates of the number of VLBW infants born in each of the contributing centres. ${ }^{6}$

Poisson regression analysis was used to estimate incidence rate ratios and hence determine the significance of trend. Fisher's exact test was used to assess relations between the infecting organism and mortality. Logistic regression was used to assess the impact of birth weight, gestational age, and organism type on mortality and overall risk of meningitis. Statistical analysis was performed using SPSS version 11.5.1 and Stata for Window (Poisson regression).

\section{RESULTS}

Overall, 3966 infants were reported with sepsis (bacteraemia and/or meningitis) in the 11 years from 1992 to 2002 inclusive. CSF examination was performed in $51 \%$ of babies The criteria for meningitis was met in 308 (7.8\%) babies.

Seventy eight $(25 \%)$ of the episodes of meningitis occurred within 48 hours of birth. This represented $9.2 \%$ of 852 infants who had early onset sepsis. Seventeen of these babies had birth weights less than $1500 \mathrm{~g}$. Table 1 lists the organisms isolated. Of the cases of EONBM, 48 (62\%) were due to GBS, and $16(21 \%)$ were due to $E$ coli. Overall, Gram negative bacilli accounted for 19 cases $(24 \%)$. Six $(8 \%)$ of the babies

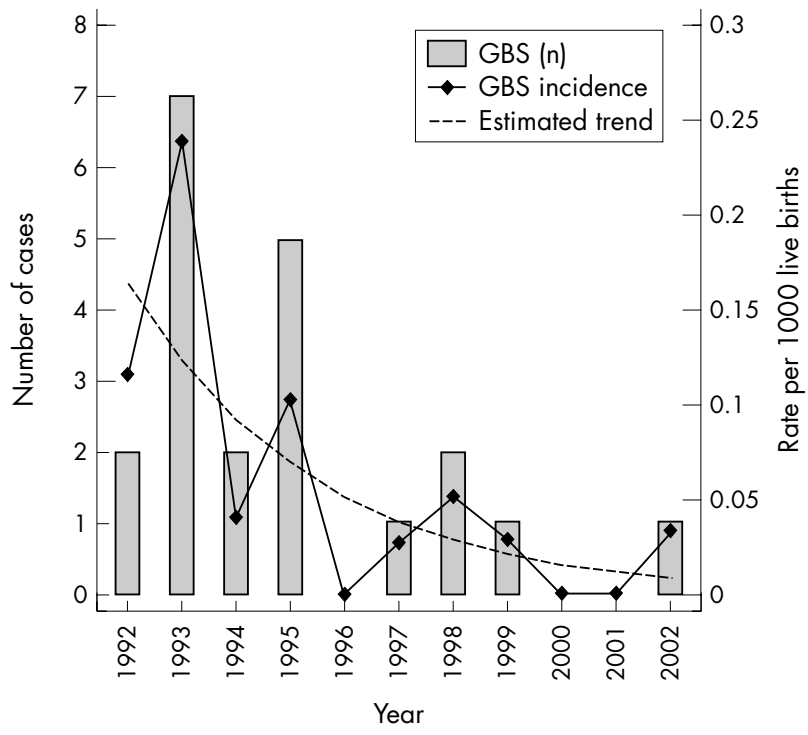

Figure 1 Early onset group B streptococcal (GBS) meningitis, Australasia, 1992-2002.

with meningitis had a positive CSF culture but a negative blood culture. CSF culture was performed in $61 \%$ of all babies with early onset sepsis. There has been no significant change with time in the proportion of babies with early onset sepsis who had a lumbar puncture performed $\left(\chi^{2}\right.$ test $\left.p=0.12\right)$.

Forty three babies with EONBM were born at the reporting hospitals (table 2), and these cases were used in the calculation of incidence rates. The overall incidence of EONBM from 1992 to 2002 was $0.11 / 1000$ live births. The rate declined from a peak of $0.41 / 1000$ in 1992 to a low of 0.06/1000 in 2001. This downward trend did not reach significance, with an estimated incident rate ratio for successive years of 0.90 (95\% confidence interval (CI) 0.81 to $1.01, \mathrm{p}=0.07$ ).

The rate of GBS meningitis fell from a peak of $0.24 / 1000$ in 1993 to no cases in 2000 and 2001 (fig 1). There was one case in 2002 (rate 0.03/1000). This downward trend was significant with an estimated incident rate ratio for successive years of 0.74 ( $95 \%$ CI 0.62 to $0.90, p=0.002$ ). When the GBS meningitis rates in the two hospitals that contributed sequential data were examined, the rate still decreased significantly with an estimated incident rate ratio of 0.66 ( $95 \%$ CI 0.46 to $0.94, \mathrm{p}=0.02$ ).

A further 45 babies had meningitis with onset between day 3 and 7 of age. If the definition of early onset meningitis was extended to include all babies less than 7 days old, there was virtually no change in the estimated incident rate ratio for EONBM, but the estimated incident rate ratio for GBS meningitis for successive years increased slightly to 0.82 , the downward trend remaining significant (95\% CI 0.71 to 0.96 , $\mathrm{p}=0.01$ ).

Table 2 Early onset neonatal bacterial meningitis for inborn babies 1992-2002

\begin{tabular}{|c|c|c|c|c|c|c|c|c|c|c|c|c|}
\hline & 1992 & 1993 & 1994 & 1995 & 1996 & 1997 & 1998 & 1999 & 2000 & 2001 & 2002 & Total \\
\hline Group B streptococcus & 2 & 7 & 2 & 5 & 0 & 1 & 2 & 1 & 0 & 0 & 1 & 21 \\
\hline Escherichia coli & 4 & 0 & 1 & 1 & 1 & 1 & 1 & 0 & 0 & 1 & 3 & 13 \\
\hline Gram negative rods & 5 & 0 & 1 & 1 & 2 & 1 & 1 & 1 & 0 & 2 & 3 & 17 \\
\hline Total in VLBW babies & 4 & 1 & 1 & 1 & 0 & 0 & 1 & 1 & 2 & 1 & 3 & 15 \\
\hline Total & 7 & 7 & 3 & 6 & 2 & 3 & 3 & 3 & 3 & 2 & 4 & 43 \\
\hline $\begin{array}{l}\text { Number of live births/year at reporting } \\
\text { hospitals }\end{array}$ & 17196 & 29434 & 50400 & 48879 & 48812 & 37691 & 38965 & 34434 & 23708 & 31838 & 30008 & 391365 \\
\hline
\end{tabular}

VLBW, Very low birth weight. 
The overall rate of $E$ coli meningitis was $0.03 / 1000$. The overall rate of Gram negative bacillary meningitis (including $E$ coli) was $0.04 / 1000$ (fig 2 ). There were no significant trends in Gram negative meningitis overall or $E$ coli meningitis in particular, with estimated incident rate ratios for successive years of 0.97 (95\% CI 0.82 to $1.15, \mathrm{p}=0.73$ ) and 0.96 (95\% CI 0.79 to $1.16, \mathrm{p}=0.67)$ respectively. There were insufficient data points for both these parameters to analyse trends in the two hospitals that contributed consecutively.

The overall rate of EONBM in VLBW infants was 1.09/1000 live births (95\% CI 0.91 to 1.27 ). There was no significant trend in the rate of meningitis in this population, with an estimated incident rate ratio for successive years of 1.00 (95\% CI 0.84 to $1.20, p=0.95)$. The most common cause of EONBM in VLBW infants was E coli $(59 \%)$.

Significant risk factors for a baby with early onset sepsis to develop meningitis were infection with GBS (16.4\% v 6.2\%; odds ratio (OR) 3.0; 95\% CI 2.3 to 3.8; p <0.0001) and infection with a Gram negative bacillus (13.7\% v 6.0\%; OR 2.5; $95 \%$ CI 2.0 to $3.2 ; \mathrm{p}<0.0001)$. Birth weight and gestational age were not independent risk factors.

Babies with early onset meningitis were more likely to die than babies with early onset sepsis without meningitis $(18.2 \% \quad v 9.8 \%$; OR $2.1 ; 95 \%$ CI 1.5 to $2.8 ; \mathrm{p}<0.0001)$. Babies with birth weight $<1500 \mathrm{~g}$ who had early onset meningitis had a significantly higher case-fatality rate $(33.6 \%)$ than babies $>1500 \mathrm{~g}$ with EONBM (6.5\%) (OR 7.2; $95 \%$ CI 4.8 to $10.9 ; \mathrm{p}<0.0001$ ). Early onset meningitis caused by Gram negative bacilli had a higher mortality than EONBM due to other organisms (28.6\% v 10.7\%; OR 3.3; 95\% CI 2.2 to 4.9; $\mathrm{p}<0.0001$ ).

Of the 852 babies with early onset sepsis, 129 (15\%) died. A lumbar puncture was not performed in $80(62 \%)$ of the 129 babies who died. Thirty two of these 80 babies were bacteraemic with GBS and 30 with $E$ coli.

Case-fatality rates for EONBM did not alter significantly over time, although absolute numbers were small. Seven (11\%) babies with EONBM died from their meningitis in the years 1992-1997, and four (24\%) babies died in the years 1998-2002 (Fisher's exact test, $\mathrm{p}=0.19$ ).

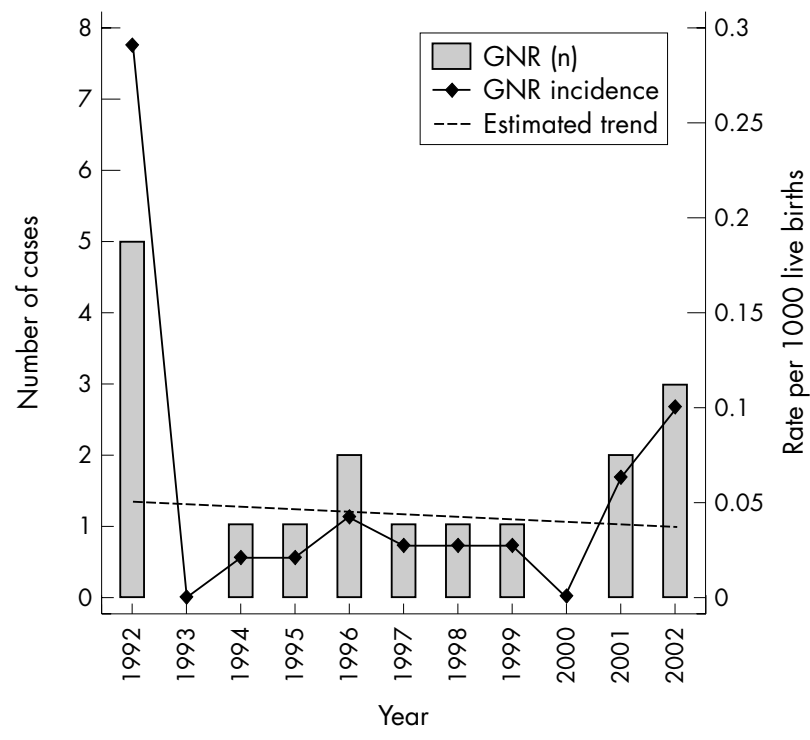

Figure 2 Early onset Gram negative bacillary (GNR) meningitis, Australasia, 1992-2002.

\section{DISCUSSION}

The major finding of our study is a significant decrease in the rate of early onset GBS meningitis over the study period. This reduction was consistent when all babies with GBS EONBM born at reporting hospitals were examined, as well as those babies born at the two centres that contributed to each year of the study. The latter finding helps to exclude bias due to irregular reporting from the various centres as the ASGNI evolved.

This reduction in GBS EONBM is further evidence of the effectiveness of maternal intrapartum antibiotic prophylaxis in preventing neonatal GBS infection. ${ }^{4-7}$ The fall in meningitis due to GBS occurred predominantly between 1993 and 1996 (fig 1). It was during these years that intrapartum antibiotic prophylaxis became more widely established in Australasia. By 1997, 11 of the maternity units involved in ASGNI had a formal policy on intrapartum antibiotic use, whereas in 1991 only three of nine had such policies. In 1997, seven of 11 units used a risk based policy, three screened for GBS at 28 weeks gestation, and one screened for GBS at 35 weeks. ${ }^{4}$ Despite this, over the 11 years of the study, GBS remained the most common cause of early onset neonatal meningitis in Australia and New Zealand, followed by E coli.

The incidence of Gram negative bacillary meningitis did not change over time in our cohort. This finding concurs with data from the ASGNI showing no recent change in overall rates of Gram negative sepsis. ${ }^{6}$ However, some recent studies in the United States have reported an increase in the incidence of Gram negative sepsis in VLBW infants, which they suggest may be attributed to widespread intrapartum antibiotic use. ${ }^{9}{ }^{10}$ It is controversial whether such changes in incidence of $E$ coli are actually due to intrapartum antibiotic prophylaxis or are coincidental. Our data show stable rates of EONBM in VLBW infants in Australasia. Although we do not have data on the antibiotic susceptibility of bacterial isolates, the static incidence of $E$ coli meningitis argues against a selection of resistant $E$ coli by intrapartum antibiotics as an explanation.

Our observed incidence of EONBM of $0.11 / 1000$ live births is lower than the rates of $0.32 / 1000$ live births ${ }^{3}$ and $0.25 / 1000$ live births ${ }^{8}$ reported in two studies from England in the late 1980s. Although the downward trend in estimated incident rate ratio did not achieve statistical significance at the 5\% level, the $95 \%$ CI of 0.81 to 1.01 suggests that a downward trend is likely.

Clinicians in ASGNI are asked to report lumbar puncture results from babies with sepsis, even if the lumbar puncture is delayed. In 1992-3, ASGNI reported that lumbar puncture was performed in only $62 \%$ of babies with proven early onset sepsis, a figure we felt was unacceptably low. ${ }^{2}$ From 19922002, a lumbar puncture was performed in only $61 \%$ of babies with early onset sepsis and in only $51 \%$ of all septic babies. The fact that there was no significant decrease in the proportion of septic babies having a lumbar puncture performed during the study period strengthens our finding of a reduction in GBS early onset meningitis. However, it is our view that this figure still represents a lack of appropriate investigation for meningitis in a significant proportion of septic babies. It has been shown that, in asymptomatic infants treated for maternal risk factors for intrapartum sepsis, a lumbar puncture does not improve treatment or outcome. ${ }^{11}$ This should not be confused with those babies who have clinical evidence of sepsis. One danger of not performing an immediate lumbar puncture in a baby with suspected sepsis is that some babies with early onset meningitis will have negative blood cultures. In our study, this proportion was $8 \%$, but was $15-30 \%$ in other studies. $^{241213}$ The practice of not performing an immediate lumbar puncture and relying solely on blood culture results 
in early onset sepsis will miss an occasional baby with meningitis. It is disturbing that $62 \%$ of babies with early onset sepsis who died from sepsis or suspected sepsis did not have a lumbar puncture performed. It is possible that some of these babies may have had undiagnosed meningitis, particularly as one of this study's findings is that GBS and Gram negative bacteraemias are significant risk factors for the development of meningitis. There are no autopsy data available to investigate this possibility. These figures suggest that all babies with proven early onset septicaemia should have a delayed lumbar puncture performed, if one was not performed immediately.

One weakness of our study is the risk of possible underreporting of cases of sepsis, as the records of the reporting neonatal units are not cross checked, and the participating ASGNI neonatologists are relied on to send complete reports. A potential source of bias is that the more academic units are probably more likely to contribute. However, any positive cases are meant to be reported. Meningitis may be underreported for infants with sepsis when a lumbar puncture was never performed. Data were not collected on antibiotic susceptibility of bacterial isolates. We are rectifying this, but have insufficient data at this time to report resistance trends. The main strength of our study is that it includes data collected over 11 years from a large multicentre cohort, enabling clear trends in epidemiology to be demonstrated. The data are reported by clinicians, and thus represent babies with clinical sepsis, rather than just positive laboratory cultures. This should minimise over-reporting of babies with false positive cultures.

Over the last 11 years the incidence of EONBM has remained constant in Australasia. There has, however, been a significant fall in the incidence of GBS meningitis. Casefatality rates remain constant, as does the incidence of meningitis caused by $E$ coli. Ongoing surveillance of neonatal infections will continue, in order to study further possible epidemiological shifts in pathogens.

\section{ACKNOWLEDGEMENTS}

Many thanks to Ms Shelley Reid for data entry, to Mr Glen Duncan for statistical advice, and to the members of ASGNI for data collection, as this surveillance would not be possible without their ongoing participation.

\section{Authors' affiliations}

M May, Department of Microbiology, The Children's Hospital at Westmead, Westmead, NSW 2145, Australia

M May, D Isaacs, Department of Immunology and Infectious Diseases, The Children's Hospital at Westmead

A J Daley, Department of Microbiology and Infectious Diseases, The Royal Children's Hospital, Parkville, Victoria 3052, Australia
S Donath, Murdoch Children's Research Institute and University of Melbourne Department of Paediatrics, Parkville, Victoria 3052, Australia

Competing interests: none declared

Australasian Study Group for Neonatal Infections consists of: C Barfield MB FRACP (Monash Medical Centre, Melbourne, Australia); D Bouchier MB FRACP (Waikato, New Zealand); G Bury MB FRACP (Hobart Hospital, Tasmania, Australia); I Bucens MB FRACP, A Ruben MB FRACP (Royal Darwin Hospital, Darwin, Australia); D Cartwright, MB FRACP (Royal Women's Hospital, Brisbane, Australia); T Clothier MBBS, J Ehrlich MB FRACP, F Morey PhD (Alice Springs Hospital, Alice Springs, Australia); B Darlow MB FRACP (Christchurch, New Zealand); S Fraser MB FRACP (Mercy Hospital, Melbourne, Australia); L Gilbert MD FRACP FRCPA (Westmead Hospital, Sydney, Australia); K Grimwood MD FRACP (Wellington, New Zealand); A Daley MB FRACP FRCPA, $P$ McDougall MD FRACP, J Royle MB FRACP (The Royal Children's Hospital, Melbourne, Australia); D Henderson-Smart MD FRACP, H Jeffery MD FRACP (King George V Hospital, Sydney, Australia); D Isaacs MD FRACP (The Children's Hospital at Westmead, Sydney, Australia); R Kohan MB FRACP (King Edward Memorial Hospital, Perth, Australia); A McPhee MB FRACP (Women's and Children's Hospital, Adelaide, Australia); R Messer MB FRACP (Cairns Base Hospital, Cairns, Australia); C Minutillo MD FRACP (Princess Margaret Hospital, Perth, Australia); D Tudehope MD FRACP (Mater Hospital, Brisbane, Australia); J Whitehall MB FRACP (Kirwan Hospital, Townsville, Australia).

\section{REFERENCES}

1 Francis BM, Gilbert GL. Survey of neonatal meningitis in Australia: 19871989. Med J Aust 1992;156:240-3.

2 Isaacs D, Barfield CP, Grimwood K, et al. Systemic bacterial and fungal infections in infants in Australian neonatal units. Med J Aust 1995; 162:198-201

3 Synnott MB, Morse DL, Hall SM. Neonatal meningitis in England and Wales: a review of routine national data. Arch Dis Child 1994:71:F75-80.

4 Isaacs D, Royle JA. Intrapartum antibiotics and early onset neonatal sepsis caused by group B Streptococcus and by other organisms in Australia. Pediatr Infect Dis J 1999;18:524-8.

5 Baltimore RS, Huie SM, Meek Jl, et al. Early-onset neonatal sepsis in the era of group B streptococcal prevention. Pediatrics 2001;108:1094-8.

6 Daley AJ, Isaacs D. Ten-year study on the effect of intrapartum antibiotic prophylaxis on early onset group B streptococcal and Escherichia coli neonatal sepsis in Australasia. Pediatr Infect Dis J 2004;23:630-4.

7 Hyde TB, Hilger TM, Reingold A, et al. Trends in incidence and antimicrobial resistance of early-onset sepsis: population-based surveillance in San Francisco and Atlanta. Pediatrics 2002;110:690-5.

8 Hristeva L, Booy R, Bowler I, et al. Prospective surveillance of neonatal meningitis. Arch Dis Child 1993;69:14-18.

9 Stoll BJ, Hansen N, Fanaroff AA, et al. Changes in pathogens causing earlyonset sepsis in very-low-birth-weight infants. N Engl J Med 2002;347:240-7.

10 Hervas JA, Ballestros F, Alomar A, et al. Increase of Enterobacter in neonatal sepsis: a twenty-two year study. Pediatr Infect Dis J 2001;20:134-40.

11 Johnson CE, Whitwell JK, Pethe K, et al. Term newborns who are at risk for sepsis: are lumbar punctures necessary? Pediatr 1997;99:10e.

12 Doctor BA, Newman N, Minich NM, et al. Clinical outcomes of neonatal meningitis in very-low-birth-weight infants. Clin Pediatr (Phila) $2001 ; 40: 473-80$

13 Franco SM, Cornelius VE. Long-term outcome of neonatal meningitis. Am J Dis Child 1992;146:567-71. 